\title{
Parnaparin: confronto con le altre eparine a basso peso molecolare
}

Lorenzo Pradelli*, Mario Eandi*

REVIEW

\begin{abstract}
Sodium parnaparin is a low molecular weight heparin (LMWH). The introduction of this drug class has represented a medical advancement in the prevention and therapy of thromboembolic pathologies, as they maintain the same efficacy of unfractionated heparin, but with simplified dosing regimens and reduced side effects.

Parnaparin has demonstrated its thromboprophylactic efficacy on both high- and moderate risk surgical patients, besides resulting effective in treating established deep vein thromboses and thrombosis-associated phlebopathies.

Alongside these clinical advantages, parnaparin and other LMWHs allow outpatient or home-based therapy of a large number of subjects that should otherwise be treated in hospital, with important savings of health resources and enhanced quality of life for the patients.

In Italy, parnaparin drug acquisition cost is the lowest among all LMWHs for the majority of their applications, permitting marginal savings in health costs at the same efficacy level.

In summary, the use of parnaparin in thrombotic pathologies has very good efficacy and safety profiles, and has positive clinical and economical outcomes for patients, health system and society as a whole.
\end{abstract}

Farmeconomia e percorsi terapeutici 2004; 5 (1): 33-45

\section{INTRODUZIONE}

L'eparina è stata introdotta nella terapia e nella prevenzione delle patologie trombotiche da circa 70 anni, contribuendo a ridurre drasticamente la morbilità da trombosi venosa profonda e l'incidenza di embolia polmonare, sua complicazione più pericolosa e causa importante di mortalità ospedaliera. Alla fine degli anni Ottanta alcuni studi clinici dimostrarono che frazioni di eparina a basso peso molecolare possedevano la medesima efficacia profilattica dell'eparina completa, ma presentavano dei vantaggi in termini di sicurezza. Oggigiorno l'utilizzo clinico delle eparine a basso peso molecolare nella terapia della trombosi venosa profonda, ma non solo, è consolidato, grazie alla superiore praticità d'impiego e tollerabilità rispetto all'eparina non frazionata, di cui mantengono l'efficacia. Lo scopo del presente articolo è di delineare un profilo farmacologico clinico e farmacoeconomico di parnaparin sodico, evidenziandone analogie e differenze rispetto alle altre eparine a basso peso molecolare.

\section{FARMACOLOGIA}

Lo sviluppo del trombo avviene nei vasi sanguigni per la costituzione di una massa solida composta da piastrine e da fibrina, nella quale restano inglobate cellule ematiche.

Il primo evento è generalmente rappresentato dall'adesione delle piastrine alla parete del vaso e dalla loro aggregazione; l'attivazione dei fattori plasmatici porta intanto alla produzione di una rete di fibrina che si estende progressivamente trattenendo emazie nel suo contesto. Nella costituzione del trombo debbono quindi essere considerate come fasi fondamentali l'adesione delle piastrine alla superficie del vaso, l'aggregazione delle piastrine fra di loro, l'attivazione della coagulazione del sangue, che ha come fenomeno finale la formazione della fibrina. Le piastrine aderiscono all'endotelio danneggiato o al subendotelio esposto dalla lesione, modificano la loro forma, manifestano i recettori per i vari attivatori, preparano la loro superficie per il coinvolgimento dei fattori coagulativi.
*Farmacologia
Clinica, Università di
Torino 
Per la concomitante attivazione dei fattori di contatto (meccanismo "intrinseco") si scatena il meccanismo coagulativo plasmatico, che si svolge attraverso le attivazioni progressive dei fattori XII, XI, IX (con la mediazione del fattore VIII) ed infine X. Più importante però è l'intervento del Fattore Tissutale, il quale, assieme al fattore VII (meccanismo "estrinseco"), accelera gli eventi coagulativi, "saltando" la prima fase della successione di attivazioni e arrivando direttamente alla stimolazione dei fattori IX e X.

La trombina promuove la trasformazione del fibrinogeno in fibrina, che avviene per polimerizzazione delle molecole di fibrinogeno.

Lo svolgimento delle reazioni piastriniche e plasmatiche è controllato da una serie di funzioni inibitorie che comprendono l'intervento della prostaciclina, degli eparani parietali, della proteina $\mathrm{C} /$ proteina $\mathrm{S}$, dell' antitrombina III e di altri inibitori delle proteasi plasmatiche.

L'eparina ha un effetto anticoagulante diretto, consistente nell' amplificazione dell' attività dell' antitrombina III, un inibitore naturale presente nel plasma, che in assenza di eparina inattiva le serinproteasi (trombina, fattori attivati X, IX, XI, XII) molto lentamente, mentre in sua presenza le blocca molto efficientemente.

L'attività anticoagulante plasmatica dell' eparina è completata da un' altra non meno importante attività antitrombotica che si svolge a livello della parete vascolare: l'eparina iniettata in circolo è captata dalle cellule endoteliali e sulla loro superficie esercita la funzione di inibizione della trombina e del fattore $\mathrm{X}$ attivato.

Le eparine a basso peso molecolare (EBPM) sono una classe di molecole ottenute per frammentazione dell'eparina naturale, o non frazionata $(\mathrm{ENF})$, che è composta di una miscela di mucopolisaccaridi dal peso molecolare che oscilla tra i 4.000 a 30.000 dalton, mentre le EBPM hanno un peso molecolare compreso tra i 4.000 e i 9.000 dalton. Il dosaggio di ENF e delle EBPM viene convenzionalmente misurato in unità internazionali di attività di inibizione del fattore $\mathrm{X}$ attivato (UI antiXa, da ora in poi solo UI), in maniera da permettere un confronto standardizzato e più semplice tra le varie molecole.

Alla frammentazione dell'eparina consegue una disgiunzione dell'attività anti trombotica da quella anticoagulativa, come indicato dal rapporto tra l'inibizione del fattore Xa e quella della coagulazione, misurato con l'aPTT. La spiegazione di questo fenomeno risiede proprio nella brevità della catena delle EBPM, che favorisce la formazione del complesso binario con antitrombina e fattore Xa rispetto al complesso ternario con la trombina: l'inibizione del fattore Xa è almeno doppia rispetto a quella della trombina. Questo rapporto viene considerato importante,

\section{Assorbimento s.c. Tmax Emivita Anti Xa/Anti IIa}

Tabella 1

Parametri

farmacocinetici di ENF

e delle EBPM

\begin{tabular}{|c|c|c|c|c|}
\hline & Assorbimento s.c. & $\operatorname{Tmax}$ & Emivita & Anti Xa/Anti IIa \\
\hline Eparina sodica & $15-30 \%$ & 3 ore & 1 ora & 1 \\
\hline Tinzaparina & $90 \%$ & 4-6 ore & 1,5 ore & 1,5 \\
\hline Dalteparina & $90 \%$ & $3-4$ ore & 4 ore & 2,6 \\
\hline Reviparina & $95 \%$ & $3-4$ ore & 3 ore & 3,5 \\
\hline Enoxaparina & $\sim 100 \%$ & 3 ore & 4,4 ore & 4 \\
\hline Nadroparina & $98 \%$ & 4-6 ore & $8-10$ ore & $>4$ \\
\hline Parnaparin & $>90 \%$ & 3 ore & 6 ore & $>4$ \\
\hline Fondaparinux & $\sim 100 \%$ & 2 ore & 17 ore & $\begin{array}{c}\text { Assenza di attività } \\
\text { anti IIa }\end{array}$ \\
\hline
\end{tabular}


in quanto gli si attribuisce un significato simile a quello dell'indice terapeutico, ossia del rapporto tra le dosi tossiche e quelle terapeutiche di una sostanza. Il rapporto antiXa/anti IIa non è esattamente sovrapponibile come concetto a quello di indice terapeutico, ma ha una certa affinità con quest'ultimo, in quanto esprime il rapporto tra le dosi necessarie ad esplicare l'azione ritenuta alla base dell'effetto indesiderato, in questo caso l'anticoagulazione con conseguente rischio di emorragia, e quelle sufficienti ad esplicare l'azione terapeutica/ profilattica, ossia l'inibizione della formazione o dell' accrescimento del trombo di fibrina.

Tale processo di accorciamento della catena prosegue ulteriormente fino ad arrivare alla creazione di molecole sintetiche, la cui struttura molecolare corrisponde alla catena più breve in grado di legare l'antitrombina III e inibire il fattore Xa. Attualmente in Italia fondaparinux è l'unico rappresentante di questa nuova classe farmacologica e il suo uso è limitato all'ambiente ospedaliero, per la prevenzione delle patologie trombotiche dopo interventi di chirurgia ortopedica ad alto rischio. L'ulteriore riduzione della lunghezza strutturale è associata alla perdita completa di attività anti IIa, ma non è stato ancora definitivamente accertato se a tale caratteristica farmacodinamica corrisponda un equivalente vantaggio clinico.

Le modificazioni strutturali che portano alla formazione delle EBPM e dei glicosamminoglicani modificano altresì le caratteristiche farmacocinetiche, rendendo più pratico il loro utilizzo clinico: l'assorbimento delle EBPM dopo somministrazione per via sottocutanea è pressoché completo e avviene in maniera prevedibile e costante, l'emivita di eliminazione plasmatica risulta allungata, anche se in maniera variabile tra le varie EPBM, permettendo comunque la monosomministrazione giornaliera. La Tabella 1 riassume le principali caratteristiche farmacologiche di ENF e delle EBPM in commercio nel nostro Paese.

Sono proprio i parametri farmacocinetici a determinare le principali differenze tra l'ENFe le varie EBPM. In particolare, l'elevata quota di farmaco assorbito e la costanza e prevedibilità della cinetica di assorbimento in seguito a somministrazione sottocutanea rendono più efficiente e agevole questa via di somministrazione per le EBPM rispetto alla ENF.

È da notare che parnaparin possiede in maniera spiccata tutte le caratteristiche che determinano la praticità d'uso delle EBPM: ottima biodisponibilità sottocutanea, lunga permanenza dell' attività anti fattore $\mathrm{Xa}$, che rimane misurabile per 20 ore dopo somministrazione di una dose singola, e un rapporto di inibizione $\mathrm{Xa} / \mathrm{II} a$ tra $\mathrm{i}$ più elevati nella classe delle EBPM [1].

La scarsa influenza di parnaparin, come delle altre EBPM, sui parametri della coagulazione comporta un ulteriore vantaggio pratico, consistente nel superamento della necessità di effettuare controlli costanti e attenti dei valori di laboratorio, indispensabili con ENF [2].

Tale caratteristica, unita all'efficienza della via di somministrazione sottocutanea, permette la gestione di molti pazienti in regime ambulatoriale, consentendo di estendere il campo applicativo dei farmaci eparinici. Essi trovano infatti utile impiego non solo nella tromboprofilassi pre- e post-operatoria, nella quale si candidano anche ad affiancare o sostituire gli anticoagulanti orali, e nella terapia della fase acuta della trombosi venosa profonda, ma anche nella terapia ambulatoriale o domiciliare di condizioni cliniche a rischio trombotico o con trombosi in atto, quali le flebiti superficiali, l'insufficienza venosa cronica e le varicoflebiti.

\section{COLLOCAZIONETERAPEUTICA}

\section{Indicazioni}

Parnaparin è indicata per la profilassi della trombosi venosa profonda in soggetti a rischio di grado moderato e alto e per la terapia delle trombosi in atto. Inoltre, è l'unica EBPM in Italia a riportare tra le indicazioni in scheda tecnica anche la terapia delle tromboflebiti acute superficiali e delle varicoflebiti, oltre che della sindrome post-flebitica e dell'insufficienza venosa cronica.

\section{Trombosi venosa profonda profilassi e terapia}

Viene definita trombosi venosa profonda (TVP) l'ostruzione, parziale o completa, di una o più vene del circolo profondo degli arti inferiori o superiori e /o delle vene della pelvi. L'embolia polmonare (EP) è la complicanza più temibile della TVP ed è causata dalla migrazione nel circolo arterioso polmonare di un trombo venoso profondo o di suoi frammenti. La TVP è un evento frequente che può complicare il decorso di interventi chirurgici, politraumatismi, malattie autoimmuni e neoplastiche, pur potendo presentarsi anche in soggetti in buone condizioni di salute. Non sono disponibili stime precise dell'incidenza della TVP, poiché essa è spesso asintomatica e di conseguenza non diagnosticata. Dati più precisi sono disponibili sulla prevalenza di TVP in alcune condizioni cliniche 
ad alto rischio. Se ricercata con la tecnica del fibrinogeno marcato, la TVP, in assenza di un'adeguata profilassi, si verifica in oltre il $10 \%$ dei pazienti di oltre 40 anni che subiscono un intervento elettivo di chirurgia addominale e in oltre il $50 \%$ di quelli sottoposti ad artroprotesi d'anca [3].

La genesi della trombosi riconosce i suoi momenti basilari negli elementi della triade di Virchow (danneggiamento della parete vasale, modificazione della corrente ematica e alterazioni ipercoagulative del sangue), per quanto oggi sia assodato che non è necessaria la partecipazione di tutti e tre gli elementi perché si abbia trombosi. Le caratteristiche individuali e le situazioni cliniche che favoriscono una o più componenti della triade sono anche i principali fattori di rischio di trombosi venosa profonda, riassunti nella Tabella 2 .

Nell' ambito delle trombosi venose profonde bisogna tenere separato il problema della prevenzione della loro insorgenza dal problema del trattamento della trombosi conclamata.

L'efficacia e la tollerabilità dell'ENF e delle EBPM nella tromboprofilassi dei pazienti sottoposti a interventi di chirurgia ortopedica, ossia del tipo di chirurgia a maggiore rischio trombotico (artroprotesi di anca o ginocchio), sono state esaminate in maniera approfondita da Eikelboom e coll. [4] in un' ampia meta-analisi, condotta sui dati ottenuti da 9 studi clinici, controllati versus placebo o assenza di trattamento, in un totale di 4.000 pazienti trattati per 4-6 settimane. L'analisi dei risultati ha rilevato che la terapia profilattica con farmaci eparinici è in grado di ridurre significativamente l'incidenza di trombosi venosa profonda sintomati-

\section{Principali fattori di rischio per TVP}

\section{Fattori predisponenti}

Età avanzata

Neoplasie maligne

Gravidanza (soprattutto $2^{\circ}$

trimestre e puerperio)

Scompenso cardiaco

Obesità

Fumo

Alterazioni ematologiche

Diabete mellito

Lupus eritematoso sistemico

e altre collagenopatie

Sindrome nefrosica

\section{Tabella2}

Principali fattori di rischio per TVP ca $(1,3 \%$ vs. $3,3 \%, \mathrm{OR}=0,38)$, di trombosi venosa asintomatica rilevata dalla venografia $(9,6 \%$ vs. $19,6 \%, \mathrm{OR}=0,48)$ e della mortalità complessiva $(0,1$ vs. $0,3 \%)$. I pazienti trattati, inoltre, hanno avuto un'incidenza di episodi emorragici maggiori inferiore a quelli dei gruppi di controllo ( $0,1 \%$ vs. $0,3 \%)$, mentre le emorragie di grado lieve-moderato sono occorse più frequentemente nei pazienti che hanno ricevuto gli anticoagulanti (3,7\% vs. $2,5 \%)$. Gli autori di questo studio hanno stimato che la profilassi a lungo termine con eparinici è in grado di evitare 20 trombosi venose profonde sintomatiche e una morte per ogni 1.000 pazienti trattati.

Le linee guida formulate dalle principali società di angiologia italiane raccomandano l'utilizzo preferenziale delle EBPM negli interventi di artroprotesi d' anca, mentre considerano ENF e EBPM farmaci di prima scelta nelle operazioni di sostituzione protesica dell'articolazione del ginocchio [5].

Per quanto riguarda parnaparin in particolare, la somministrazione quotidiana di $6400 \mathrm{UI}$ a pazienti ad alto rischio trombotico per la profilassi post-operatoria dopo chirurgia ortopedica o vascolare, in cui l'incidenza attesa di TVP era compresa tra 50 e $70 \%$, è stata in grado di limitare la percentuale di pazienti colpiti a valori oscillanti tra l' $1 \%$ e il $20 \%$ [2].

Negli studi condotti su pazienti a rischio trombotico di grado minore, in quanto sottoposti a interventi di chirurgia generale, in cui l'incidenza attesa di TVP in assenza di profilassi è compresa tra il $15 \%$ e il $30 \%$, la somministrazione giornaliera di 3200 UI di parnaparin è risultata in grado di ridurre l'incidenza di TVP a valori compresi tra lo 0 e il $6,5 \%$, dunque significativamente minori $[6,7]$.

In un ampio studio clinico condotto per valutare l'efficacia di due schemi posologici di parnaparin nella prevenzione dell'embolismo polmonare in pazienti sottoposti a interventi di chirurgia generale, in cui la mortalità da embolia prevista era dell'1\%, la somministrazione di 3200 o 6400 UI di parnaparin è risultata associata a un'incidenza di embolismo polmonare (confermato dal Rxtorace o dalla scintigrafia polmonare) di $0,6 \% \mathrm{e}$ $0 \%$, rispettivamente. In questo studio, l'efficacia di parnaparin nella prevenzione dell' embolismo polmonare è risultata almeno uguale a quella dell'ENF, somministrata a dosi di 10.000 o $15.000 \mathrm{U}$ [1].

In letteratura è disponibile un unico studio di tromboprofilassi post-chirurgica con parnaparin controllato vs. placebo: l'incidenza di TVP è risultata nulla nel gruppo di pazienti sottoposti a trattamento con $3200 \mathrm{UI} /$ die di 
parnaparin, mentre nei pazienti trattati con il placebo di controllo tale valore è stato del $6 \%$ [6].

Diversi studi hanno invece confrontato parnaparin all'ENF nella profilassi della TVP in pazienti con differenti livelli di rischio. In tutti questi studi $[2,3,8]$ parnaparin, alle dosi di 3200 o $6400 \mathrm{UI} / \mathrm{die}$, ha dimostrato una tendenza alla superiorità rispetto a ENF, somministrata alle dosi di 10.000 o 15.000 unità/die, senza tuttavia raggiungere una superiorità statisticamente significativa, in parte per la ridotta dimensione dei campioni esaminati (50-140 pazienti).
Un ampio studio condotto con i medesimi schemi posologici, ma su un campione più ampio, composto da 610 pazienti, ha invece dimostrato la superiorità statistica di parnaparin rispetto all'eparina non frazionata nella tromboprofilassi post-chirurgica: la diagnosi di TVP è stata posta nel 3,2\% dei pazienti trattati con parnaparin, a fronte di un $6,3 \%$ riscontrato nel gruppo dell'ENF [1]. I risultati di questo studio hanno inoltre rilevato una minor incidenza di embolismo polmonare con il trattamento con parnaparin $(0,3 \%$ vs. $1 \%)$, per quanto tale differenza non sia risultata statisticamente significativa.

\begin{tabular}{|c|c|c|c|c|c|c|}
\hline Bibliografia & $\begin{array}{l}\text { Tipo di } \\
\text { chirurgia }\end{array}$ & Posologia & $\begin{array}{l}\text { Pazienti } \\
\text { valutati }\end{array}$ & $\begin{array}{l}\text { Tecnica di } \\
\text { conferma }\end{array}$ & $\begin{array}{c}\text { TVP } \\
\text { confermate } \\
(\%)\end{array}$ & $\begin{array}{l}\text { Effica cia } \\
\text { relativ a }\end{array}$ \\
\hline \multicolumn{7}{|c|}{ Confronti versus placebo (PI) } \\
\hline 6 & $\begin{array}{l}\text { Generale/ } \\
\text { addominale }\end{array}$ & $\begin{array}{l}\text { P: } 3200 \mathrm{UI} \text { uid } \\
\mathrm{Pl}\end{array}$ & $\begin{array}{l}50 \\
50\end{array}$ & $\begin{array}{l}\text { Ecodoppler/ } \\
\text { venografia }\end{array}$ & $\begin{array}{l}0 \\
6\end{array}$ & $\mathrm{P}>\mathrm{P1}^{*}$ \\
\hline \multicolumn{7}{|c|}{ Confronti versus eparina non frazionata (E NF) } \\
\hline 2 & Ortopedica & $\begin{array}{l}\text { P: } 3200 \text { UI bid } \\
\text { ENF: } 5000 \text { UI tid }\end{array}$ & $\begin{array}{l}70 \\
70\end{array}$ & Ecodoppler & $\begin{array}{l}7,1 \\
10\end{array}$ & $\mathrm{P} \geq \mathrm{ENF}$ \\
\hline 8 & Addominale & $\begin{array}{l}\text { P: } 3200 \text { UI uid } \\
\text { ENF: } 5000 \text { UI tid }\end{array}$ & $\begin{array}{l}45 \\
45\end{array}$ & $\begin{array}{l}\text { Venografia/ } \\
\text { ecodoppler/ } \\
\text { test di captazione } \\
\text { del fibrinogeno }\end{array}$ & $\begin{array}{c}0 \\
2,2\end{array}$ & $\mathrm{P} \geq \mathrm{ENF}^{*}$ \\
\hline 9 & Ortopedica & $\begin{array}{l}\text { P: } 3200 \text { UI bid } \\
\text { ENF: } 5000 \text { UI tid }\end{array}$ & $\begin{array}{l}68 \\
68\end{array}$ & Ecodoppler & $\begin{array}{l}1,5 \\
2,9\end{array}$ & $\mathrm{P} \geq \mathrm{ENF}^{*}$ \\
\hline 10 & Ortopedica & $\begin{array}{l}\text { P: } 3200 \text { UI bid } \\
\text { ENF: } 5000 \text { UI tid }\end{array}$ & $\begin{array}{l}25 \\
24\end{array}$ & Venografia & $\begin{array}{l}20 \\
29\end{array}$ & $\mathrm{P} \geq \mathrm{ENF}$ \\
\hline 3 & Vascolare & $\begin{array}{l}\text { P: } 6400 \text { UI uid } \\
\text { ENF: } 5000 \text { UI bid }\end{array}$ & $\begin{array}{l}46 \\
46\end{array}$ & Ecodoppler & $\begin{array}{l}6,5 \\
8,6\end{array}$ & $\mathrm{P} \geq \mathrm{ENF}^{*}$ \\
\hline 11 & $\begin{array}{l}\text { Generale/ } \\
\text { addominale }\end{array}$ & $\begin{array}{l}\text { P: } 3200 \text { UI uid } \\
P: 6400 \text { UI uid } \\
\text { ENF: } 5000 \text { UI bid } \\
\text { ENF: } 5000 \text { UI tid }\end{array}$ & $\begin{array}{c}250 \\
58 \\
134 \\
168\end{array}$ & $\begin{array}{l}\text { Ecodoppler/ } \\
\text { pletismografia/ } \\
\text { vemografia }\end{array}$ & $\begin{array}{l}3,6 \\
1,7 \\
5,2 \\
7,1\end{array}$ & $\mathrm{P} \geq \mathrm{ENF}$ \\
\hline
\end{tabular}

Legenda: uid monosomministrazione giomalier a; bid due somministrazioni al giomo; tid tre somministrazioni al giorno; $P>E N F$ superiorità preventiva di pamaparin statisticamente significativa $(p<0,05)$;

$P \geq E N F$ equivalenza o superiorità (non stat isticamente signific ativa) preventiva di parnaparin;

$P \geq E N F^{*}$ equivalenza o superiorità di parnaparin (non testata per significatività statistica)

Tabella 3

Prevenzione della TVP in chirurgia: studi clinici controllati (Modificata da Frampton \& Faulds, 1994) 
Non sono invece disponibili studi di confronto diretto tra parnaparin e altre EBPM, né nella profilassi della TVP né in altre indicazioni; questa lacuna si ricontra piuttosto frequentemente nell'ambito di questa classe farmacologica, dove gli studi di confronto diretto tra le varie molecole sono veramente esigui, tanto che diversi Autori [12] già da tempo ne hanno sollecitato la progettazione e conduzione. Per quanto è possibile evincere da comparazioni indirette, tuttavia, non sembrano esistere differenze clinicamente significative tra le varie EBPM in termini di efficacia profilattica [13].

Il ruolo dei farmaci eparinici nel trattamento della TVP in atto è ben definito, in virtù della dimostrata efficacia in termini di miglioramento degli esiti emodinamici e clinici e di prevenzione delle recidive. La terapia di prima scelta della TVP prevede l'uso combinato di anticoagulanti orali e ENF o EBPM, con sospensione del farmaco eparinico al raggiungimento dell' INR target $(2,0-3,0)$, per la maggior parte dei pazienti, riservando il trattamento trombolitico ai pazienti giovani e a quelli con embolismo polmonare grave [14].

Le EBPM hanno un'efficacia paragonabile all'ENF nella terapia della TVP, ma il loro avvento ha determinato una notevole semplificazione del trattamento. La terapia con ENF, infatti, richiede la permanenza del paziente in ospedale per la somministrazione endovenosa del farmaco e il monitoraggio continuo dei parametri coagulativi, mentre le EBPM consentono un regime ambulatoriale, grazie all'efficienza della somministrazione sottocutanea e alla scarsità di effetti sull'aPTT, che rende superfluo il costante controllo dei valori di laboratorio $[15,16]$. Numerosi studi hanno dimostrato che gli esiti delle trombosi venose profonde a medio e a lungo termine (3-12 mesi) sono almeno altrettanto favorevoli dopo terapia domiciliare con EBPM che dopo ENF endovenosa in infusione continua, con un incidenza paragonabile, ma spesso inferiore, di complicanze emorragiche [17-22]. La possibilità di ricorrere alla terapia a domicilio, con significative riduzioni della durata della degenza ospedaliera, non è solamente teorica, come dimostrato da Segal et al. [23] in una recentissima meta-analisi, i cui risultati indicano che l'uso delle EBPM per la terapia della TVP è associato a tempi di ricovero medi significativamente più brevi $(2,7$ gg vs. 6,5$)$ che con ENF, a parità di efficacia e sicurezza.

Per quanto riguarda la prevenzione secondaria delle recidive di TVP dopo un episodio sintomatico, diversi studi clinici indicano che le EBPM possono anche venire utilizzate in alternativa alla terapia anticoagulante orale nei casi di riconosciuta controindicazione a questi agenti o di loro difficile gestione.

Gonzalez-Fajardo e coll. [24] hanno condotto uno studio con controllo venografico per confrontare l' efficacia e la tollerabilità dei due approcci terapeutici alternativi su 165 pazienti con TVP. Il primo gruppo ha ricevuto un trattamento con ENF e anticoagulanti orali, con dosaggio aggiustato in base agli esami di laboratorio, mentre il secondo gruppo ha ricevuto enoxaparina, somministrata per due volte al dì nella prima settimana, in regime di ricovero ospedaliero, e in unica somministrazione giornaliera per 90 giorni dopo la dimissione.

I risultati del confronto hanno messo in evidenza che la terapia domiciliare con EBPM della fase post-acuta delle TVP è significativamente più efficace della terapia con anticoagulanti cumarinici, sia in termini di regressione del trombo, come evidenziata alla venografia (score di Marder: $-49,4 \%$ vs. $-24,5 \%$, $\mathrm{p}<0,001)$, sia in termini di recidive sintomatiche $(9,5 \%$ vs. $23,7 \%$, p < 0,05). La terapia con EBPM è inoltre risultata associata a un rischio significativamente inferiore di complicanze emorragiche $(1,1 \%$ vs. $10 \%$, p < 0,05$)$, per quanto tale riduzione sia stata determinata interamente da emorragie minori.

Uno studio analogo, condotto su oltre 150 pazienti con TVP per un periodo compreso tra i 3 e i 6 mesi, ha utilizzato un'altra EBPM, nadroparina, ottenendo risultati simili. Nel corso dei 12 mesi di osservazione, nel gruppo trattato con EBPM si sono verificate meno recidive di TVP, meno emorragie gravi e meno decessi, per quanto le differenze non siano apparse significative dal punto di vista statistico. Significativamente inferiore, invece, è risultata l'incidenza di reflusso venoso nelle vene comunicanti rispetto al gruppo che ha ricevuto EBPM [25].

\section{Insufficienza venosa cronica}

L'insufficienza venosa cronica (IVC) è una condizione clinica conseguente ad uno scompenso del funzionamento delle vene periferiche, in cui il ritorno del sangue venoso verso il cuore non è garantito né in posizione ortostatica, né in clinostatismo. Si distinguono un'insufficienza del sistema venoso superficiale, una del sistema venoso profondo, o una condizione mista, con inadeguatezza di entrambi.

La patogenesi delle manifestazioni soggettive e obiettive dell' IVC è condizionata dall'ipertensione venosa, localizzata o diffusa, con 
ripercussioni emoreologiche sulla macro e microcircolazione: l'edema ne costituisce la manifestazione caratteristica, sia sul piano fisiopatologico che clinico.

L'IVC colpisce prevalentemente il sesso femminile fino alla quinta-sesta decade, mentre a età superiori non si notano significative differenze fra sessi. La prevalenza dell'IVC a carico degli arti inferiori è del 10-50\% nella popolazione adulta maschile e del $50-55 \%$ in quella femminile. La malattia varicosa è presente, clinicamente manifesta, nel 10-33\% delle donne e nel 10-20\% dei maschi adulti [26-28].

I due quadri clinici più caratteristici dell' insufficienza venosa profonda e superficiale sono la sindrome post-flebitica e la varicoflebite, rispettivamente.

La sindrome post-flebitica è la sequela evolutiva di una TVP e si presenta con un caratteristico quadro clinico (edema, senso di pesantezza all'arto colpito, anomalie di pigmentazione ed eventuali ulcere da stasi), dovuto alle alterazioni dell'emodinamica venosa conseguenti all'ostruzione cronica del circolo venoso profondo, ma soprattutto alla incontinenza delle valvole del circolo venoso profondo e delle vene perforanti, a sua volta causata dalla ricanalizzazione spontanea delle vene ostruite.

L'utilità di parnaparin nella gestione delle complicanze acute della sindrome post-flebitica (infiammazione e/o ostruzione) è stata valutata in uno studio clinico condotto da Verardi S e coll [29] su 77 pazienti e controllato vs. ENF. I risultati hanno messo in evidenza che il trattamento con parnaparin, somministrato per via sottocutanea alla dose di 6400 UI per i primi 10 giorni e a dosaggio dimezzato nei successivi 50, è associata ad significativi miglioramenti dei parametri clinici (dolore, edema, ulcerazioni, ecc.) e dei parametri emodinamici misurati con il Doppler.

La forma clinica più frequente di processo flebitico superficiale è, senza dubbio, la varicoflebite, che insorge sia su varici essenziali che su quelle secondarie a IVC, con meccanismo patogenetico legato alla stasi venosa nel tratto dilatato e contorto.

Il trombo neoformato aderisce rapidamente alla parete venosa, per la presenza di una vivace risposta infiammatoria, e ciò rappresenta uno dei motivi della rarità di eventi embolici nei casi di tromboflebite superficiale.

La clinica di una varicoflebite è caratterizzata da esordio rapido, con aumento della consistenza, colorito rossastro, calore e dolore spontaneo esacerbato dalla palpazione.

Il trattamento più comune delle varicoflebiti, considerate patologie acute di modesta gravi- tà nonostante alcuni studi indichino un rischio di sviluppare TVP fino nel $25 \%$ dei casi [30], prevede l'applicazione locale di FANS e l'utilizzo di metodi di contenzione elastica per contrastare l'infiammazione e la stasi venosa. Nei casi in cui sussiste il pericolo di una propagazione del processo trombotico alle vene del circolo venoso profondo, per le dimensioni o per la posizione anatomica del trombo $(<3 \mathrm{~cm}$ dallo sbocco) o per altri fattori di rischio concomitanti, è utile un trattamento antitrombotico con eparine; tra quelle disponibili in Italia, parnaparin è l'unica EBPM a riportare in scheda tecnica l'indicazione al trattamento delle tromboflebiti acute superficiali, per quanto anche altre vengano prescritte in questa indicazione, di carattere prettamente ambulatoriale, in cui l'ENF ha chiari svantaggi dal punto di vista della praticità d'impiego.

L'utilità di parnaparin nel trattamento delle varicoflebiti è stata valutata in studi condotti su pazienti affetti da flebopatie e sindromi associate di diversa natura e gravità. Il dosaggio di parnaparin utilizzato in queste ricerche è dipeso dal tipo e dalla gravità della patologia trattata, ma lo schema di mantenimento tipico prevedeva la somministrazione di 4250 o 6400 $\mathrm{UI} /$ die, per un periodo oscillante tra 1 e 3 mesi. In generale, la terapia con parnaparin ha determinato un miglioramento sintomatico, confermato dalle misure di funzionalità e da test emodinamici, che anche per l'esiguità del campione non è sempre risultato statisticamente significativo [1]. Nei confronti diretti che hanno coinvolto pazienti affetti da varicoflebiti (Tabella 4), l'efficacia di parnaparin è risultata simile a quella di ENF.

Riassumendo, gli orientamenti più moderni prevedono dunque una terapia antitrombotica con EBPM nei pazienti con tromboflebite superficiale che interessi il distretto venoso della coscia, che rischi di estendersi al circolo venoso profondo, o che presentino altri fattori di rischio trombotico [3133].

\section{TOLLERABILITÀ}

Tra gli effetti collaterali indesiderati che comporta l'uso di eparina sono di particolare rilievo le complicanze emorragiche, direttamente legate al meccanismo d' azione della moleco1a. Nella letteratura scientifica sulle eparine, le emorragie vengono abitualmente divise in ematomi localizzati nella sede d'iniezione del farmaco e in emorragie in altre sedi.

L'incidenza di ematomi nella sede d'inoculo è risultata più bassa con parnaparin che con $\mathrm{ENF}$, anche quando i risultati sono stati corret- 


\begin{tabular}{|c|c|c|c|c|}
\hline \multirow[t]{2}{*}{ Diagnosi } & \multirow[t]{2}{*}{ Posologia } & \multicolumn{2}{|c|}{ Risultati } & \multirow[t]{2}{*}{ Efficacia relativa } \\
\hline & & $\begin{array}{l}\text { Parametri emodinamici } \\
\text { (\% variazione) }\end{array}$ & Sintomi & \\
\hline SPF & $\begin{array}{l}\text { P: } 4250 \text { UI/die per } 90 \mathrm{gg}(24 \mathrm{pz}) \\
\text { ENF: } 10000 \mathrm{UI} / \text { die per } 90 \mathrm{gg}(22 \mathrm{pz})\end{array}$ & $\begin{array}{l}\uparrow \text { FVM }(8)^{*} \\
\downarrow \text { PVTP }(12)^{*} \\
\uparrow \text { FVM }(3) \\
\downarrow \text { PVTP }(11)^{*}\end{array}$ & $\begin{array}{l}\downarrow \text { Edema*, cianosi, prurito*, distrofia } \\
\text { cutanea*, dolore* } \\
\downarrow \text { Edema*, cianosi, prurito*, distrofia } \\
\text { cutanea*, dolore* }\end{array}$ & $\mathrm{P}=\mathrm{ENF}$ \\
\hline IVC & $\begin{array}{l}\text { P: } 4250 \text { UI/die per 90gg ( } 35 \mathrm{pz}) \\
\text { ENF: } 15000 \text { UI/die per } 90 \mathrm{gg}(35 \mathrm{pz})\end{array}$ & $\begin{array}{l}\downarrow \mathrm{CV} 40 \text { (5)Miglioramento FV in } 15 \mathrm{pz} \\
\downarrow \mathrm{CV} 40 \text { (4)Miglioramento FV in } 12 \mathrm{pz}\end{array}$ & $\begin{array}{l}\downarrow \text { Edema*, dolore*, cianosi*, } \\
\text { ipertermia locale*, parestesie* } \\
\uparrow \text { Funzione arto* } \\
\downarrow \text { Edema*, dolore*, cianosi*, } \\
\text { ipertermia locale*,parestesie* } \\
\uparrow \text { Funzione arto* }\end{array}$ & $\mathrm{P}=\mathrm{ENF}$ \\
\hline $\begin{array}{l}\text { TVP, } \\
\text { SPF, } \\
\text { TF, VF }\end{array}$ & $\begin{array}{l}\text { P: } 3200,6400 \text { o } 12800 \mathrm{UI} / \mathrm{die} \text { per } 60 \mathrm{gg}(30 \mathrm{pz}) \\
\text { ENF:12500-25000 UI/die per } 60 \mathrm{gg}(30 \mathrm{pz})\end{array}$ & $\begin{array}{l}\downarrow \operatorname{EVP}(23) \\
\downarrow \mathrm{SVP}(18) \\
\downarrow \mathrm{EVP}(20) \\
\downarrow \mathrm{SVP}(18)\end{array}$ & $\begin{array}{l}\downarrow \text { Edema, cianosi, dolore } \\
\uparrow \text { Edema, cianosi, dolore }\end{array}$ & $\mathrm{P}=\mathrm{ENF}$ \\
\hline $\begin{array}{l}\text { FSA, } \\
\text { TVP, VF }\end{array}$ & $\begin{array}{l}\text { P: } 6400 \text { UI/die per } 28-42 \text { gg ( } 32 \text { pz) } \\
\text { ENF: } 25000 \text { UI per } 28-42 \text { gg ( } 32 \mathrm{pz})\end{array}$ & $\begin{array}{l}\text { Normalizzazione flusso sanguigno in } 10 \mathrm{pz} \\
\text { Normalizzazione flusso sanguigno in } 7 \mathrm{pz}\end{array}$ & $\begin{array}{l}\downarrow \text { Edema*, dolore*, ipertermia locale* } \\
\downarrow \text { Funzione arto* } \\
\uparrow \text { Edema*, dolore*, ipertermia locale* } \\
\downarrow \text { Funzione arto* }\end{array}$ & $\mathrm{P}=\mathrm{ENF}$ \\
\hline SPF, VF & ENF: $20000 \mathrm{UI} /$ die per $10 \mathrm{gg}+12500 \mathrm{UI} / \mathrm{die}$ per $=50 \mathrm{gg}$ & $\begin{array}{l}\downarrow \text { EVP }(17)^{*} \\
\downarrow \text { SVP }(31)^{*} \\
\uparrow \text { VPRTex }(130)^{*} \\
\uparrow \text { Fasicità }(18)^{*} \\
\downarrow \text { EVP }(13)^{*} \\
\downarrow \text { SVP }(28)^{*} \\
\uparrow \text { VPRTex }(130)^{*} \\
\uparrow \text { Fasicità }(20)^{*}\end{array}$ & $\begin{array}{l}\downarrow \text { Edema* }^{*} \text {, dolore*, ipertermia locale* } \\
\text { e rash* }\end{array}$ & $\mathrm{P}=\mathrm{ENF}$ \\
\hline
\end{tabular}

Legenda: SPF sindrome post-flebitica; IVC insufficienza venosa cronica; TVP trombosi venosa profonda; TF tromboflebite; VF varicoflebite; FSA flebite superficiale acuta; FVM flusso venoso massimale; PVTP pressione venosa tibiale posteriore; CV40 capacitanza venosa (con manicotto a $40 \mathrm{mmHg}$ ); FV flusso venoso; EVP pressione venosa in ortostatismo; SVP pressione venosa in clinostatismo; VPRTex tempo di recupero pressione venosa dopo esercizio; P parnaparin; ENF eparina non frazionata; * $\mathrm{P}<0,05$ vs basale

11 Tabella 4

Terapia delle flebopatie e dell'IVC: studi clinici controllati vs. eparina (Modificata da Frampton \& Faulds, 1994) 
ti per compensare la minore frequenza di somministrazione [34]. Nel più ampio degli studi di confronto, condotto su 610 pazienti, l'incidenza di ematomi nella sede d'iniezione è risultata del 5\% nei pazienti trattati con parnaparin (3.200-6.400 UI/die), a fronte del $23 \%$ osservato nei pazienti che avevano ricevuto ENF (10.000-15.000 UI/die) [1].

Per quanto riguarda le emorragie in altre sedi, ferita chirurgica compresa, l'incidenza con parnaparin appare simile o inferiore a quella osservata con ENF [1]. Nel più ampio degli studi condotti con parnaparin, emorragie clinicamente evidenti sono avvenute nell' $1 \%$ dei pazienti, contro il $4 \%$ dei pazienti del gruppo di confronto, trattati con ENF; nello stesso lavoro, il $2 \%$ e 1' $8 \%$ dei pazienti che hanno ricevuto parnaparin e ENF, rispettivamente, hanno sviluppato ematoma della ferita chirurgica $[1,11]$.

Tra le complicanze non emorragiche è da tenere presente la possibilità di reazioni allergiche e la comparsa di trombocitopenia: una trombocitopenia modesta può esser rilevata nel $5 \%$ dei pazienti trattati con ENF, compare precocemente ed appare in relazione all'influsso aggregante sulle piastrine posseduto dall' eparina; una trombocitopenia grave è molto più rara, compare più tardivamente (dopo 410 giorni) ed è in connessione alla formazione di aggregati piastrinici, mediati da meccanismo immunologico.

Le eparine a basso peso molecolare presentano un minor effetto sulle piastrine e sembrano coinvolte molto più raramente nella comparsa delle trombocitopenie gravi. Negli studi clinici con parnaparin non si sono osservati casi di trombocitopenia, ma la possibilità che si verifichino non è da escludere totalmente, anche in relazione alla segnalazione di alcuni casi associati all'uso di altre EBPM.

\section{ASPETTI ECONOMICI}

L'elevata prevalenza della TVP nei pazienti sottoposti a chirurgia comporta un significativo impatto economico, oltre che clinico, per i sistemi sanitari di tutto il mondo. La costo-efficacia degli interventi profilattici nei soggetti a rischio trombotico è ampiamente dimostrata, sia nei confronti della rinuncia alla profilassi, sia rispetto al monitoraggio, seguito da terapia in caso di conferma di trombosi.

La costo-efficacia relativa delle EBPM rispetto all'ENF è stata valutata in diversi studi, sia nella profilassi che nella terapia della TVP. Dall'insieme di queste valutazioni emerge chiaramente che la gestione ambulatoriale di una buona percentuale di pazienti, resa pos- sibile dalle caratteristiche farmacologiche delle EBPM, è in grado di indurre un netto risparmio di risorse sanitarie.

In uno studio farmacoeconomico condotto sulla base dei risultati di un ampio trial clinico secondo le prospettive del terzo pagante statunitense e canadese, la terapia con EBPM è risultata dominante rispetto alla terapia con ENF, ossia associata contemporaneamente a migliori esiti clinici ed economici, nonostante il superiore costo per l'acquisto del farmaco [35].

Il medesimo risultato è stato ottenuto in uno studio farmacoeconomico effettuato da Estrada e coll. [36], che hanno modellizzato gli esiti clinici e i costi sanitari di un'ipotetica coorte di 1.000 pazienti con TVP prossimale, trattati in una stazione di terapia intensiva e seguiti per tre mesi. Tre sono state le strategie alternative simulate: 1) la somministrazione endovenosa di ENF; 2) la terapia sottocutanea con EBPM $(40 \%$ a domicilio, $60 \%$ in ospedale); 3) un approccio misto, con somministrazione di ENF ai pazienti ricoverati e di EBPM a quelli trattati a casa. I risultati del modello hanno rivelato che le strategie EBPM e ENF/EBPM dominano l'alternativa ENF (cioè producono migliori risultati clinici a un costo economico inferiore) nello scenario di base e nella maggioranza delle simulazioni effettuate nel corso dell' analisi di sensibilità. In particolare, la strategia a base di EBPM diviene economicamente più conveniente della somministrazione di ENF quando la percentuale di pazienti trattati a domicilio supera il 14\%, valore sempre realizzabile nella pratica clinica [36].

Per quanto la gestione domiciliare delle patologie trombotiche presenti indubbi vantaggi per la società e per il paziente, vi sono alcuni aspetti che vale la pena sottolineare.

Una prima questione riguarda la percentuale e le caratteristiche dei pazienti che possono essere gestiti, almeno parzialmente, al di fuori dell'ospedale. Generalmente, gli studi indicano che tale percentuale si assesta intono all' $80 \%$ [37], in quanto non sono eligibili per il trattamento ambulatoriale o domiciliare i pazienti ad alto rischio emorragico, le donne in gravidanza, $\mathrm{i}$ bambini e i nefropatici [38].

Un secondo aspetto cruciale, dal quale dipende la possibilità di trasformare i risparmi da potenziali a effettivi, riguarda la disponibilità territoriale di strutture e servizi sanitari che permettano il trattamento a casa del paziente $o$ in ambulatorio, oltre a garantire un' adeguata e tempestiva assistenza in caso 
di necessità. Per sfruttare appieno il potenziale risparmio dovuto alla minor durata della degenza ospedaliera di questi pazienti, inoltre, è necessario che il personale e le risorse liberate vengano impiegate in maniera efficiente; ciò probabilmente richiede una certa riorganizzazione delle strutture sanitarie.

Un terzo aspetto riguarda la ridistribuzione dell'onere economico tra i vari attori coinvolti, cioè paziente, ospedale, $\mathrm{SSN}$ e società nel complesso. La dimissione precoce dopo chirurgia e la terapia domiciliare delle TVP rappresentano indubbiamente occasioni di risparmio per l'ospedale e, di riflesso, per il SSN, per quanto quest'ultimo debba comunque coprire i costi dei farmaci. Per il paziente, si potrebbe invece verificare un certo aumento dei costi non sanitari, per la necessità di numerosi spostamenti tra domicilio e luogo di cura o di procurarsi personale sanitario per la somministrazione sottocutanea, ma tali svantaggi sono presumibilmente ampiamente compensati dal miglioramento della qualità di vita. Alcuni pazienti, tuttavia, potrebbero percepire in maniera negativa la dimissione precoce e/o la terapia domiciliare/ambulatoriale, sentendosi "abbandonati", per cui da un punto di vista etico sarebbe opportuno coinvolgere il paziente nella decisione sul tipo di trattamento da effettuare.

Per la società nel complesso le conseguenze economiche del passaggio da gestione ospedaliera con ENF alle nuove forme di trattamento possibili con EBPM sono indubbiamente positive, grazie alla riduzione dei costi sanitari, associata a miglioramento degli esiti clinici e della qualità di vita dei cittadini.

\section{COSTIFARMACEUTICI}

In assenza di evidenze della differente efficacia o sicurezza delle varie EBPM, esse vanno considerate terapeuticamente equivalenti. In questi casi, l' analisi costo-efficacia può essere validamente sostituita da una più semplice analisi di minimizzazione dei costi. I costi da considerare sono unicamente quelli farmaceutici, in quanto si presuppone che, a parità di risultati terapeutici e di sicurezza, le altri voci di costo si equivalgano.

La Tabella 5 riporta le confezioni e i prezzi delle EBPM disponibili in Italia, mentre in Tabella 6 è indicato il costo d'acquisto del farmaco necessario per un ciclo terapeutico al prezzo praticato al pubblico. Le posologie valoriz-

\begin{tabular}{|c|c|c|c|}
\hline F arma co & Confezione & $\begin{array}{l}\text { Prezzo } \\
\text { (Euro) }\end{array}$ & $\begin{array}{c}\text { Prezzo/unità } \\
\text { (Euro) }\end{array}$ \\
\hline $\begin{array}{l}\text { Parnaparin } \\
\text { (Fhrum) }\end{array}$ & $\begin{array}{l}6 \text { fiale-siringa } 3200 \mathrm{UI} \\
6 \text { fiale-siringa } 4250 \mathrm{UI} \\
6 \text { fiale-siringa } 6400 \mathrm{UI}\end{array}$ & $\begin{array}{l}21,56 \\
28,76 \\
38,25\end{array}$ & $\begin{array}{l}3,59 \\
4,79 \\
6,38\end{array}$ \\
\hline $\begin{array}{l}\text { Enoxaparina } \\
\text { (Clex ane-Clex ane } \mathrm{T})\end{array}$ & $\begin{array}{l}6 \text { fiale-siringa } 2000 \mathrm{UI} \\
6 \text { fiale-siringa } 4000 \mathrm{UI} \\
2 \text { fiale-siringa } 6000 \mathrm{UI} \\
2 \text { fiale-siringa } 8000 \mathrm{UI} \\
2 \text { fiale-siringa } 10000 \mathrm{UI}\end{array}$ & $\begin{array}{l}21,04 \\
39,91 \\
17,04 \\
19,35 \\
22,81\end{array}$ & $\begin{array}{l}3,51 \\
6,65 \\
8,52 \\
9,68 \\
11,41\end{array}$ \\
\hline $\begin{array}{l}\text { Reviparina } \\
\text { (C livarina) }\end{array}$ & $\begin{array}{l}10 \text { fiale-siringa } 1750 \mathrm{UI} \\
10 \text { fiale-siringa } 4200 \mathrm{UI} \\
1 \text { flacone } 42000 \mathrm{UI}\end{array}$ & $\begin{array}{l}29,49 \\
75,17 \\
73,10\end{array}$ & $\begin{array}{c}2,95 \\
7,52 \\
73,10\end{array}$ \\
\hline $\begin{array}{l}\text { Dalteparina } \\
\text { (Fragmin) }\end{array}$ & $\begin{array}{l}6 \text { fiale-siringa } 2500 \mathrm{UI} \\
6 \text { fiale-siringa } 5000 \mathrm{UI} \\
4 \text { fiale siringa } 15000 \mathrm{UI}\end{array}$ & $\begin{array}{l}20,36 \\
36,46 \\
76,80\end{array}$ & $\begin{array}{c}3,39 \\
6,07 \\
19,20\end{array}$ \\
\hline $\begin{array}{l}\text { Nadroparina } \\
\text { (Fraxiparina) }\end{array}$ & $\begin{array}{l}6 \text { fiale-siringa } 2850 \mathrm{UI} \\
6 \text { fiale-siringa } 3800 \mathrm{UI} \\
10 \text { fiale-siringa } 5700 \mathrm{UI} \\
10 \text { fiale-siringa } 7600 \mathrm{UI}\end{array}$ & $\begin{array}{l}21,48 \\
29,01 \\
64,02 \\
72,67\end{array}$ & $\begin{array}{l}3,58 \\
4,84 \\
6,40 \\
7,27\end{array}$ \\
\hline $\begin{array}{l}\text { Fondaparimux } \\
\text { (Arixtra) }\end{array}$ & $\begin{array}{l}2 \text { fiale-siringa } 2,5 \mathrm{mg} \\
10 \text { fiale-siringa } 2,5 \mathrm{mg} \\
20 \text { fiale-siringa } 2,5 \mathrm{mg}\end{array}$ & $\begin{array}{c}31,56 \\
156,79 \\
313,58\end{array}$ & $\begin{array}{l}15,78 \\
15,68 \\
15,68\end{array}$ \\
\hline
\end{tabular}


zate in termini monetari sono quelle segnalate sulla scheda tecnica dei farmaci per le principali indicazioni autorizzate, per il paziente tipo di $70 \mathrm{~kg}$. A questo proposito, è utile sottolineare che le 3 unità posologiche di parnaparin disponibili in commercio coprono le necessità profilattiche e terapeutiche di tutti i soggetti, in quanto parnaparin non necessita di aggiustamenti posologici in base al peso corporeo, a differenza di molte altre EBPM. La congruenza dell'unità posologica e del numero di dosi per confezione è tutt'altro che secondaria dal punto di vista economico e clini$\mathrm{co}$, in quanto la necessità di calcolare il dosaggio in base al peso e la possibile esigenza di utilizzare solo parzialmente le fiale possono condurre ad errori di dosaggio e sprechi.

Come evidenziato in Tabella 6 , il costo di acquisto di parnaparin è il più basso tra le EBPM disponibili in Italia per la profilassi antitrombotica dei soggetti ad alto rischio e per la terapia della TVP in atto. La forbice tra il prezzo di parnaparin e quello di altre EBPM si apre ancor più per soggetti di peso elevato, in considerazione della necessità di adeguare la posologia.

\section{CONCLUSIONI}

Parnaparin sodico è una eparina a basso peso molecolare. L'introduzione di questa classe farmaceutica rappresenta un progresso della medicina nell'ambito della prevenzione e della terapia delle patologie trombotiche, permettendo di mantenere l'efficacia dell'eparina non frazionata con uno schema posologico semplificato e una riduzione degli effetti indesiderati.

Parnaparin, infatti, al pari delle altre EBPM può essere efficacemente somministrata per via sottocutanea in una o due prese giornaliere, senza necessità di ricorrere all' infusione endovenosa utilizzata con eparina non frazionata. Parnaparin ha dimostrato la sua efficacia nella profilassi antitrombotica in chirurgia, sia su pazienti ad alto rischio che in quelli con un profilo di rischio più favorevole ed è inoltre efficace nella terapia delle trombosi venose profonde già in atto e nelle flebopatie associate a trombosi, risultando l'unica EBPM ad avere l'ufficialità di queste ultime indicazioni in Italia. Oltre ai vantaggi clinici, parnaparin, come altre EBPM, permet-

\begin{tabular}{|c|c|c|c|c|}
\hline \multirow[t]{2}{*}{ F arma co } & & \multicolumn{3}{|c|}{ Indicazione } \\
\hline & & Profil. TVP MR (10 gg) & Profil. TVPAR (30 gg) & Terap ia TVP (10 gg) \\
\hline \multirow{3}{*}{$\begin{array}{l}\text { Parnaparin } \\
\text { (Fhuxum) }\end{array}$} & Posologja & $10 \mathrm{flsir} .3200 \mathrm{UI}$ & $30 \mathrm{fl}$ sir. $4250 \mathrm{UI}$ & $20 \mathrm{fl}$ sir. $6400 \mathrm{UI}$ \\
\hline & Costo/unità & 3,59 E/fiala & 4,79 E/fiala & 6,38 Efiala \\
\hline & Costo ciclo & 35,93 Euro & 143,80 Euro & 127,50 Euro \\
\hline \multirow[t]{3}{*}{$\begin{array}{l}\text { Enoxaparina } \\
\text { (Clexane - Clexane } T)\end{array}$} & Posologja & 10 flsir. $2000 \mathrm{UI}$ & $30 \mathrm{fl}$ sir $4000 \mathrm{UI}$ & $\begin{array}{r}10 \mathrm{fl} \text { sir. } 8000 \mathrm{UI}+ \\
10 \mathrm{fl} \text { sir. } 6000 \mathrm{UI}\end{array}$ \\
\hline & Costo/unità & 3,51 E/fiala & 6,65 E/fiala & $9,68-8,52 \mathrm{E} /$ fiala \\
\hline & Costo ciclo & 35,06 Euro & 199,55 Euro & 181,95 Euro \\
\hline \multirow[t]{3}{*}{$\begin{array}{l}\text { Reviparina } \\
\text { (C livarina) }\end{array}$} & Posologia & $10 \mathrm{flsir} .1750 \mathrm{UI}$ & $30 \mathrm{fl}$ sir $4200 \mathrm{UI}$ & $\begin{array}{r}3 \text { flac } 42,000 \mathrm{UI}+ \\
20 \text { siringhe }\end{array}$ \\
\hline & Costo/unità & 2,95 E/fiala & 7,52 E/fiala & $73,10 \mathrm{E} /$ tlac \\
\hline & Costo ciclo & 29,49 Euro & 225,51 Euro & 219,13 Euro + siringhe \\
\hline \multirow{3}{*}{$\begin{array}{l}\text { Dalteparina } \\
\text { (Fragmini) }\end{array}$} & Posologia & $10 \mathrm{flsir} .2500 \mathrm{UI}$ & $30 \mathrm{fl}$ sir $5000 \mathrm{UI}$ & 10 fl. Sir 15000 UI \\
\hline & Costo/unità & 3,39 E/fiala & 6,07 E/fiala & $19,20 \mathrm{E}$ /fiala \\
\hline & Costo ciclo & 33,93 Euro & 182,30 Euro & 192,00 Euro \\
\hline \multirow[t]{3}{*}{$\begin{array}{l}\text { Nadroparina } \\
\text { (Fraxiparina) }\end{array}$} & Posologia & $10 \mathrm{flsir} .2850 \mathrm{UI}$ & $\begin{array}{c}2 \mathrm{fl} . \operatorname{sir} 5700 \mathrm{UI}+ \\
28 \mathrm{fl} 3800 \mathrm{UI}\end{array}$ & $20 \mathrm{fl}$ sir. $7600 \mathrm{UI}$ \\
\hline & Costo/unità & 3,58 E/fiala & $6,4-4,84$ E/fiala & 7,27 E/fiala \\
\hline & Costo ciclo & 35,80 Euro & 148,18 Euro & 145,34 Euro \\
\hline \multirow{3}{*}{$\begin{array}{l}\text { Fondaparinux } \\
\text { (Arixtra) }\end{array}$} & Posologia & Non indicato & $30 \mathrm{fl} \mathrm{sir} 2,5 \mathrm{mg}$ & Non indicato \\
\hline & Costo/unità & & 15,68 E/fiala & \\
\hline & Costo ciclo & & 470,37 Euro & \\
\hline
\end{tabular}

Legenda: MR moderato rischio; $A R$ alto risc hio; TVP trombosi venosa profonda

\section{Tabella 6}

Costi farmaceutici delle EBPM alle dosi segnalate in scheda tecnica per le varie indicazioni 
te la gestione ambulatoriale o domiciliare di un gran numero di pazienti che dovrebbero essere trattati in ospedale, consentendo notevoli risparmi di risorse sanitarie e un miglioramento della qualità di vita del paziente stesso.

Il costo d'acquisto di parnaparin è il più basso tra le EBPM disponibili in Italia per la maggior parte delle indicazioni, per cui il suo utilizzo induce un risparmio di costi sanitari, a parità di efficacia con le altre molecole di questa classe.

In conclusione, l'utilizzo di parnaparin nelle malattie trombotiche è caratterizzato da un ottimo profilo di efficacia e di tollerabilità, con risvolti clinici ed economici favorevoli per il paziente, il sistema sanitario nazionale e la società.

\section{BIBLIOGRAFIA}

1. Frampton JE, Faulds D. Parnaparin. A review of its pharmacology, and clinical application in the prevention and treatment of thromboembolic and other vascular disorders. Drugs. 1994 Apr;47(4):652-76.

2. Chiapuzzo E, Orengo GB, Ottria G, Chiapuzzo A, Palazzini E, Fusillo M. The use of low molecular weight heparins for postsurgical deep vein thrombosis prevention in orthopaedic patients. J Int Med Res. 1988 Sep-Oct;16(5):359-66.

3. Speziale F, Verardi S, Taurino M, Nicolini G, Rizzo L, Fiorani P, Palazzini E. Low molecular weight heparin prevention of post-operative deep vein thrombosis in vascular surgery. Pharmatherapeutica. 1988;5(4):261-8.

4. Eikelboom JW, Quinlan DJ, Douketis JD. Extended-duration prophylaxis against venous thromboembolism after total hip or knee replacement: a meta-analysis of the randomised trials. Lancet. 2001 Jul 7;358(9275):9-15.

5. SIAPAV (Società Italiana di Angiologia e Patologia Vascolare) Linee guida per la diagnosi e il trattamento della trombosi venosa profonda- disponibile sul sito web http://www.siapav.it/ita/start.html

6. Valle I, Sola G, Origone A. Controlled clinical study of the efficacy of a new low molecular weight heparin administered subcutaneously to prevent post-operative deep venous thrombosis. Curr Med Res Opin. 1988;11(2):80-6.

7. Pellegrino A, Balta D, De Girolamo C, Di Ceglie F, Pappalettera F, et al. Prevention of post-surgical deep vein thrombosis in urology. Prophylactic use of a new ow molecular weight heparin. Clinical Trials Journal 1988;25:16471.

8. Garcea D, Martuzzi F, Santelmo N, Savoia M, Casertano MG, Furno A, Ruggeri V. Post-surgical deep vein thrombosis prevention: evaluation of the risk/benefit ratio of fractionated and unfractionated heparin. Curr Med Res Opin. 1992;12(9):572-83.

9. Mascali F, Condorelli A, Salanitri G, Palazzini E, Iani P, et al. Postsurgery thromboembolism prevention by LMW heparin subcutaneous administration. European Review for Medical and Pharmaceutical Sciences 1988;10:135-41.

10. Pini M, Tagliaferri A, Manotti C, Lasagni F, Rinaldi E, Dettori AG. Low molecular weight heparin (Alfa LHWH) compared with unfractionated heparin in prevention of deep-vein thrombosis after hip fractures. Int Angiol. 1989 Jul-Sep;8(3):134-9.

11. Verardi S, Casciani CU, Nicora E, Forzano F, Origone A, Valle I, Catania G, Salanitri G, Salcuni P, Azzarone M, et al. A multicentre study on LMW-heparin effectiveness in preventing postsurgical thrombosis. Int Angiol. 1988 JulSep;7(3 Suppl):19-24.

12. Davidson BL. Differentiation of low molecular weight heparins in treatment of acute deep vein thrombosis. Semin Thromb Hemost. 1999;25 Suppl 3:107-12.

13. Lassen MR. Comparative efficacy of low-molecular-weight heparins in orthopedic surgery. Semin Thromb Hemost. 2000;26 Suppl 1:53-6.

14. Ageno W. Treatment of venous thromboembolism. Thromb Res. 2000 Jan 1;97(1):V63-72.

15. Hylek EM, Regan S, Henault LE, Gardner M, Chan AT, Singer DE, Barry MJ. Challenges to the effective use of unfractionated heparin in the hospitalized management of acute thrombosis. Arch Intern Med. 2003 Mar 10;163(5):621-7. 
16. MacFarlane LL, Weart CW. An Introduction to Low-Molecular-Weight Heparins and Their Use in the Treatment of Deep Venous Thrombosis. Am J Ther. 1995 Aug;2(8):569-580.

17. Grau E, Tenias JM, Real E, Medrano J, Ferrer R, Pastor E, Selfa S. Home treatment of deep venous thrombosis with low molecular weight heparin: Long-term incidence of recurrent venous thromboembolism. Am J Hematol. 2001 May;67(1):10-4.

18. Davidson BL. DVT treatment in 2000: state of the art. Orthopedics. 2000 Jun;23(6 Suppl):s651-4.

19. Manganaro A, Giannino D, Lembo D, Bruni F, Consolo F. Evolution in the pharmacological treatment of venous thrombosis according to evidence-based medicine. Minerva Cardioangiol. 2000 Dec;48(12 Suppl 1):41-51.

20. Schmidt C. Outpatient treatment of acute deep venous thrombosis of the lower limbs Ann Cardiol Angeiol (Paris). 2002 Jun;51(3):152-7.

21. Belcaro G, Nicolaides AN, Cesarone MR, Laurora G, De Sanctis MT, Incandela L, Barsotti A, Corsi M, Vasdekis S, Christopoulos D, Lennox A, Malouf M. Comparison of low-molecular-weight heparin, administered primarily at home, with unfractionated heparin, administered in hospital, and subcutaneous heparin, administered at home for deep-vein thrombosis. Angiology. 1999 Oct;50(10):781-7.

22. Labas P, Ohradka B, Vladimir J, Cambal M. The home treatment of deep vein thrombosis with low molecular weight heparin, forced mobilisation and compression. Int Angiol. 2000 Dec;19(4):303-7.

23. Segal JB, Bolger DT, Jenckes MW, Krishnan JA, Streiff MB, Eng J, Tamariz LJ, Bass EB. Outpatient therapy with low molecular weight heparin for the treatment of venous thromboembolism: a review of efficacy, safety, and costs. Am J Med. 2003 Sep;115(4):298-308.

24. Gonzalez-Fajardo JA, Arreba E, Castrodeza J, Perez JL, Fernandez L, Agundez I, Mateo AM, Carrera S, Gutierrez V, Vaquero C. Venographic comparison of subcutaneous low-molecular weight heparin with oral anticoagulant therapy in the long-term treatment of deep venous thrombosis. J Vasc Surg. 1999 Aug;30(2):283-92.

25. Lopez-Beret P, Orgaz A, Fontcuberta J, Doblas M, Martinez A, Lozano G, Romero A. Low molecular weight heparin versus oral anticoagulants in the long-term treatment of deep venous thrombosis. J Vasc Surg. 2001 Jan;33(1):77-90.

26. Callam MJ. Epidemiology of varicose veins. Br J Surg. 1994 Feb;81(2):167-73.

27. Cesarone MR, Belcaro G, Nicolaides AN, Laurora G, De Sanctis MT, Incandela L, Barsotti A. Epidemiology and costs of venous diseases in central Italy. The San Valentino Venous Disease Project. Angiology. 1997 Jul;48(7):583-93

28. Cesarone MR, Belcaro G, Nicolaides AN, Geroulakos G, Griffin M, et al. 'Real' epidemiology of varicose veins and chronic venous diseases: the San Valentino Vascular Screening Project. Angiology. 2002 Mar-Apr;53(2):119-30.

29. Verardi S, Ippoliti A, Pistolese GR. Antithrombotic treatment during acute inflammatory complications of patients affected by postphlebitic syndrome: LMW-heparin versus standard heparin. Int Angiol. 1988 Jul-Sep;7(3 Suppl):33-40

30. Guex JJ. Thrombotic complications of varicose veins. A literature review of the role of superficial venous thrombosis. Dermatol Surg. 1996 Apr;22(4):378-82.

31. Superficial Thrombophlebitis Treated By Enoxaparin Study Group. A pilot randomized double-blind comparison of a low-molecular-weight heparin, a nonsteroidal anti-inflammatory agent, and placebo in the treatment of superficial vein thrombosis. Arch Intern Med. 2003 Jul 28;163(14):1657-63

32. Bounameaux H, Reber-Wasem MA. Superficial thrombophlebitis and deep vein thrombosis. A controversial association. Arch Intern Med. 1997 Sep 8;157(16):1822-4.

33. Kalodiki E, Nicolaides AN. Superficial thrombophlebitis and low-molecular-weight heparins. Angiology. 2002 Nov-Dec;53(6):659-63

34. Gruttadauria G, Palazzini E. Open study of the medical treatment of postphlebitic syndromes with a new molecular weight heparin: parnaparin. Progress Reports 1993;5:13-20.

35. Hull RD, Pineo GF, Raskob GE The economic impact of treating deep vein thrombosis with low-molecular-weight heparin: outcome of therapy and health economy aspects. Haemostasis. 1998;28 Suppl 3:8-16.

36. Estrada CA, Mansfield CJ, Heudebert GR. Cost-effectiveness of low-molecular-weight heparin in the treatment of proximal deep vein thrombosis. J Gen Intern Med. 2000 Feb;15(2):108-15.

37. Bossuyt PM, Prins MH. Does low-molecular-weight heparin reduce the costs of venous thromboembolism treatment? Haemostasis. 2000;30 Suppl 2:136-40.

38. Lindmarker P. Can all patients with deep vein thrombosis receive low-molecular-weight heparin in an outpatient setting? Haemostasis. 1999 Dec;29 Suppl S1:84-8. 